\title{
Aquel lejano Oeste: paradojas de la (in)movilidad en la ciudad de Santa $\mathrm{Fe}$
}

That Far West: paradoxes of (im)mobility in the city of Santa Fe

Estefanía Szupiany

\begin{abstract}
Resumen
El crecimiento desigual y fragmentado del borde Oeste de la ciudad de Santa Fe puede ser analizado en función de la movilidad e inmovilidad allí experimentada. Una paradoja materializada en el trazado de la Avenida Circunvalación en tanto espacio destinado a las altas velocidades $y$, al mismo tiempo, en tanto formalización de un límite y barrera al intercambio socio-espacial. Con el objetivo de revisar los fundamentos epistemológicos de las políticas urbanas que derivaron en omisiones y resoluciones tecnocráticas, este artículo propone recuperar la historicidad del proceso de ideación y planificación, a través de los diferentes documentos y planes urbanísticos confeccionados para la ciudad, enfocando el análisis en los modos en que fue concebido al margen Oeste en relación al Río Salado.
\end{abstract}

Palabras clave: movilidad; inmovilidad; desigualdad socio-espacial; límites urbanos, Santa Fe.

\begin{abstract}
The uneven and fragmented growth of the western edge of the city of Santa Fe, in Argentina, can be analysed in terms of the mobility and immobility experienced there. A paradox materialized in the design of the belt highway as a space destined to high speeds and, at the same time, as a limit and a barrier to socio-spatial exchange. With the aim of reviewing the epistemological foundations of urban policies (that led to omissions and technocratic resolutions), this paper tries to recover the historicity of the ideation and planning process through different documents and urban plans made to the city, analyzing the ways the west bank was conceived in relation to the Salado river.
\end{abstract}

Keywords: mobility; immobility; socio-spatial inequality; urban boundaries, Santa Fe. 


\section{Introducción}

La ciudad de Santa Fe, capital de la provincia homónima ubicada en la región Centro-Este de la República Argentina, representa el centro administrativo, funcional y de servicios con mayor jerarquía del continuum urbano denominado Gran Santa Fe. Con una población total de 490.171 habitantes, según Censo Nacional de Población, Hogares y Viviendas 2010, su crecimiento se originó desde el extremo sureste, en vinculación con el trazado fundacional y el curso de la Laguna Setúbal, hacia el Norte y el Oeste. En relación a esta última direccionalidad, la mancha urbana avanzó a lo largo del siglo XX sobre terrenos anegadizos pertenecientes a los bañados del Río Salado, generando una creciente vulnerabilidad social y espacial profundizada en las últimas décadas. Por otro lado, un patrón territorial de crecimiento responde a la prolongación de vías circulatorias en distintas direcciones, incluyendo en su extensión a las localidades vecinas de Monte Vera y Recreo al Norte; Santo Tomé y Sauce Viejo al Suroeste; San José del Rincón y Arroyo Leyes al Este (Figura 1).

Figura 1 - Ciudad de Santa Fe y localidades próximas: continuum urbano Gran Santa Fe

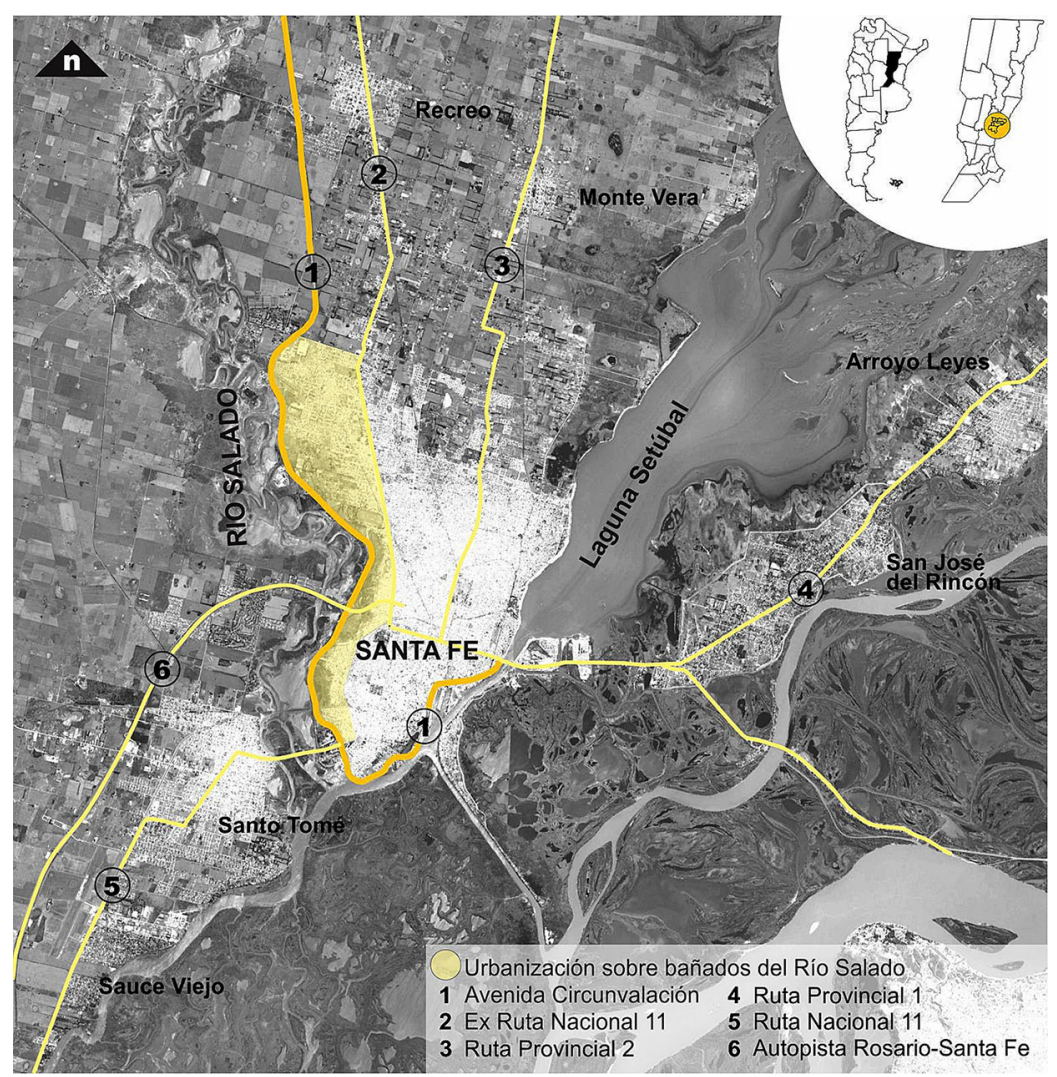

Fuente: elaboración propia a partir de imagen satelital Google Earth. 
Una rápida interpretación de estos crecimientos puede argumentarse en función de su emplazamiento sobre la intersección de los ríos Salado y Paraná. No obstante, la exploración de lecturas más complejas y pormenorizadas de estos procesos orientó la presente investigación al estudio de dinámicas urbanas vinculadas a patrones de movilidad dominantes. En este sentido, es posible comprender los distintos crecimientos mencionados a partir, por un lado, del aumento progresivo de la automovilidad y el impulso de un modelo expansivo en torno a las principales vías de comunicación vial; por otro, a partir de procesos de continuidad de la mancha urbana en dirección Norte y Oeste vinculados con patrones de movilidad dependientes, fundamentalmente, del sistema de transporte público, con cierto grado de dificultad para abastecer la creciente demanda. La consolidación de ambas tendencias, sujetas a determinados medios de locomoción, se convirtió en factor determinante de la aceleración de procesos fragmentarios y de la territorialización de la desigualdad socio-espacial. Al mismo tiempo que puede haber inmovilidad creciente por congestión, afirma Gutiérrez (2009), puede haber inmovilidad creciente por aislamiento e insularidad. En adelante, se entenderá esta disparidad con el par movilidad - inmovilidad en tanto modo de aproximación a la problemática abordada.

En el marco de esta mirada centrada en las movilidades urbanas el interrogante que motiva el presente trabajo se vincula con la intervención de las políticas urbanas en las diversas movilidades e inmovilidades que construyen territorios desiguales y fragmentados. Evitando reproducir enfoques reduccionistas que asimilan movilidad y transporte, los que traen aparejadas acciones superficiales e imposturas políticas, resulta imprescindible abordar la problemática desde marcos teóricos-metodológicos que complementan el campo de conocimiento tradicional del transporte. Recientemente, un grupo de teorías y abordajes provenientes de las ciencias sociales inauguraron un nuevo paradigma para el estudio de la movilidad. Este mobility turn propone superar la separación entre lo social y lo técnico hacia una perspectiva socio-tecnológica, perspectiva de abordaje retomada aquí con el objetivo de revisar los fundamentos epistemológicos de las políticas urbanas. Para ello, se propone enfocar el análisis en el caso particular del crecimiento sobre los bañados del Río Salado hacia el Oeste de la ciudad de Santa Fe, y su interacción con políticas urbanas fundadas en una histórica omisión y en resoluciones tecnocráticas, materializadas éstas últimas en la construcción de la Avenida Circunvalación. Se plantea, por lo tanto, examinar la movilidad e inmovilidad experimentada en el borde Oeste desde análisis retrospectivos del proceso de ideación y planificación de la ciudad, a través de los diferentes documentos urbanísticos y planes urbanos que, más allá de su formalización y concreción, alimentaron los modos de pensar, concebir y accionar en la ciudad. En definitiva, se trata de recuperar la historicidad del pensamiento subyacente a las políticas urbanas que acompañaron el crecimiento desigual y fragmentado del borde Oeste.

A continuación, la presentación del trabajo se estructura en tres apartados principales. En primer lugar, se introduce el caso de estudio examinando el par 
movilidad-inmovilidad en tanto conflicto allí experimentado. En el segundo apartado, se reflexiona en torno a los fundamentos epistemológicos que subyacen a las políticas de movilidad urbana, a la luz de los estudios contemporáneos enmarcados en el mobility turn. En tercer lugar, se analiza la problemática en el caso de estudio propuesto a partir de la reconstrucción del proceso de ideación urbana, en función de los distintos planes confeccionados para la ciudad de Santa Fe a lo largo del siglo XX.

\section{Las infraestructuras viales en perspectiva: el caso de la Avenida Circunvalación Oeste}

El crecimiento de la mancha urbana hacia el Oeste de la ciudad de Santa Fe presenta hoy un límite bien definido materializado en la Avenida Circunvalación, un límite entendido aquí más allá de su conformación física-espacial y, por consiguiente, en tanto límite al intercambio socio-espacial. El trazado de esta vía de alcance nacional se originó en las primeras obras de defensa construidas a la vera del Río Salado. Hacia finales de la década de 1930, un doble proceso impulsó el crecimiento urbano en dirección Oeste y la consecuente ocupación de terrenos anegadizos; por un lado, el incremento del valor inmobiliario que recibieron los suelos próximos al puerto de ultramar, por otro, el crecimiento demográfico experimentado en la ciudad a partir de la llegada de migrantes rurales. La rentabilidad disminuía hacia el Oeste y, rápidamente, el crecimiento se extendió más allá de las vías ferroviarias sobre los bañados del Río Salado. La generación de una situación vulnerable y su posterior agravamiento derivó en la implementación de políticas urbanas tendientes a controlar el curso fluvial, materializadas en la construcción del terraplén Irigoyen. No obstante, esta obra de singular importancia ingenieril para la ciudad fue concebida en términos estrictamente técnicos, alejada de los planteamientos higienistas y de embellecimiento que por entonces surgieron en la ciudad. En este sentido, el proceso de ocupación de los bañados del Oeste se mantuvo escindido de la planificación e ideación urbana, profundizando un estado de vulnerabilidad ante la escasez de servicios urbanos, nulas restricciones de ocupación, y la reproducción de condiciones marginales. Décadas más tarde, su carácter meramente protector se convierte además en contenedor de un tránsito incompatible con la dinámica urbana, a partir de la construcción de la Avenida Circunvalación Oeste. Construidos sus dos primeros tramos entre los años 1996 y 1998 y concluida en 2012 luego de la catástrofe hídrica del año 2003, ${ }^{1}$ esta obra vial vino a resolver una demanda funcional a nivel local y, fundamentalmente, regional y nacional, en tanto nuevo eje de circulación de la Ruta Nacional 11 (Figura 2).

Ahora bien, resueltos ambos requerimientos funcionales, es decir la consolidación de las defensas hídricas en la escala local y la ampliación del espacio destinado a las altas velocidades y la rápida circulación en las escalas superiores, cabe el interrogante acerca de la relación emergente entre la Avenida Circunvalación y el tejido urbano adyacente. En este sentido, y con el objetivo de examinar dicha relación, una 
Figura 2 - Etapas de concreción de la Avenida Circunvalación Oeste

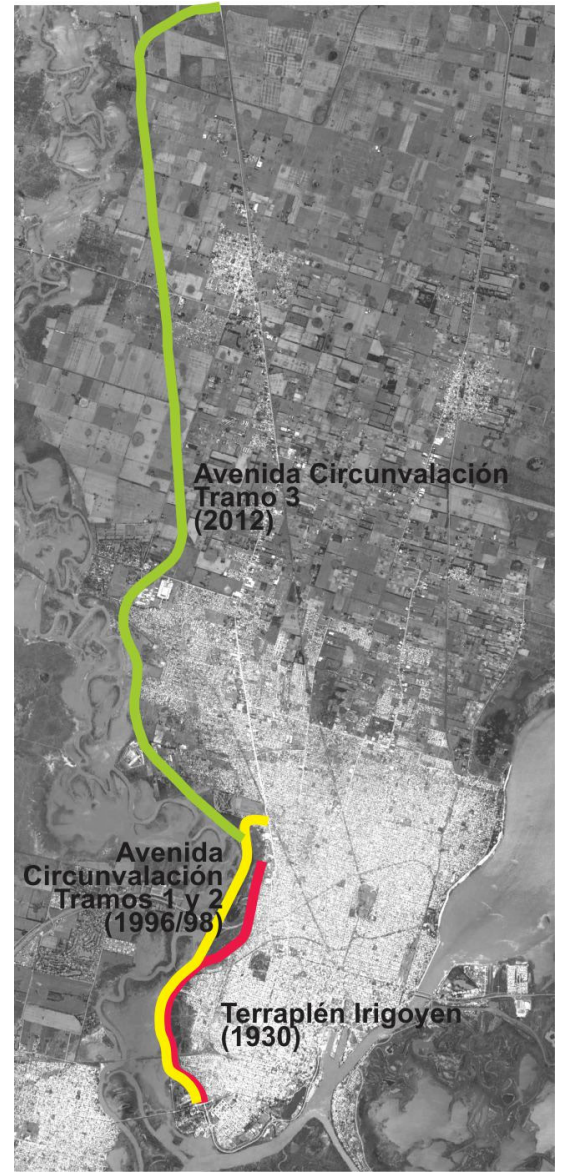

Fuente: elaboración propia a partir de imagen satelital Google Earth.

primera aproximación a la prensa local puede resultar esclarecedora para la construcción de fundamentos críticos al modo en el que se insertan las infraestructuras viales en el medio urbano $y$, por lo tanto, a las políticas urbanas en torno a la movilidad. Una lectura compleja puede hacerse en referencia a aquellos titulares periodísticos relacionados con hechos recientes acontecidos en la Circunvalación, a saber, "Circunvalación
Oeste: 10 minutos de alto riesgo" (El Litoral, 27/6/2012); "Vandalismo contra autos en la Circunvalación Oeste y la autopista a Rosario" (El Litoral, 3/10/2014); "Le tiraron un palo en la circunvalación para robarle la moto" ( LT10, 7/4/2017); "Fuego en la Circunvalación Oeste" (El Litoral, 14/8/2017); "Reiterados ataques a los automóviles en la Ruta n70" (El Litoral, 5/9/2017). A partir de estos acontecimientos y, fundamentalmente, a partir de la 
interpretación y comunicación de los mismos por parte de los medios, puede ampliarse el debate en torno a la relación infraestructura - vida urbana y, precisamente, a la dinámica del borde Oeste de la ciudad de Santa Fe, en función de las siguientes reflexiones vinculadas al problema de la movilidad:

- La interpretación de los conflictos que alteran el normal funcionamiento de la vía circulatoria, principalmente en lo que respecta a la seguridad vial y a la rápida circulación, se traducen en la reproducción de "obstáculos peligrosos" y en "riesgos para los conductores", relacionados en su mayoría con la cotidianeidad de los barrios cercanos a la Circunvalación. Trabajadores dependientes del uso de la bicicleta como medio de transporte, carros recolectores de basura, "cirujas de la zona", animales sueltos - perros, caballos, vacas -, humo ocasionado por la quema de pastizales; "piedrazos" originados por "vándalos de la zona". Connotaciones que posicionan a los conductores y a los habitantes de aquellos barrios en tanto víctimas y victimarios, respectivamente, reproduciendo una imagen estigmatizada y denostada de aquellos barrios del Oeste.

- El predominio del carácter funcional y de servicio por sobre el potencial paisajístico de la ribera Oeste, deviene en la materialización de la Avenida Circunvalación en tanto borde y límite urbano. Su emplazamiento a "espaldas" del Río Salado puede interpretarse como el desaprovechamiento de una oportunidad a la integración urbana de los barrios del Oeste, si se piensa en la posibilidad de convertir los bañados del Salado en espacios públicos de uso colectivo destinados al intercambio de grupos sociales y a la revalorización de un sector urbano postergado desde siempre. En definitiva, lo único que importa aquí es circular, lo más rápido posible y sin mirar hacia los costados. Resulta, por lo tanto, una movilidad creciente para unos, en contraste con una creciente inmovilidad relativa para otros (Gutierrez, 2009).

Ambas reflexiones sugieren la necesaria incorporación de las infraestructuras viales en el marco de la discusión contemporánea construida en torno al concepto de movilidad urbana; al tiempo que suscitan una aproximación histórica a los procesos fragmentarios que dieron lugar a la construcción marginal del borde Oeste. Definido así el objeto de investigación, la relación entre la Avenida Circunvalación y el tejido urbano de los barrios adyacentes, se propone aquí examinar dicha interacción a partir de las políticas urbanas concernientes a la problemática de la movilidad y la inmovilidad; analizando el proceso de ideación y planificación urbana a través de los diferentes documentos urbanísticos y planes urbanos confeccionados para la ciudad de Santa Fe. Documentos que, según Novick (2012), "[...] condensan el estado del conocimiento sobre la cuestión urbana en un momento dado" (p. 35); y, por su parte, Collado (1994) sostiene: "El proyecto en la ciudad moviliza personas e ideas, replantea los datos disponibles, construye un horizonte de deseos y articula las expectativas sociales, constituyendo un momento de síntesis" (p. 3). En este sentido, y según se busca hipotetizar, la conformación contemporánea del borde Oeste y su vinculación con el trazado de la Avenida Circunvalación responde a un proceso de construcción desigual, donde las políticas urbanas se redujeron a resoluciones 
tecnocráticas alejadas de los ideales urbanos que alimentaron la planificación de la ciudad de Santa Fe a lo largo del siglo XX. Ante el paradigma racional y funcionalista, que aún subyace en el urbanismo local, se entiende que el camino hacia la integración urbana requiere de nuevas perspectivas que aborden los problemas urbanos en términos socio-espaciales $y$, precisamente, los problemas en torno a la movilidad e inmovilidad urbana que incluyen no solo a los medios de trasporte sino también a los sujetos y a los espacios de la movilidad.

\section{De las infraestructuras viales a los espacios de la movilidad: repensando los fundamentos epistemológicos de las políticas de movilidad urbana}

En primer lugar, se torna necesario definir qué se entiende por movilidad y qué la diferencia del transporte; en este sentido, Gutiérrez (2012) sostiene que recrear los conceptos permite revisar las mediciones, las evaluaciones $y$, lo que aquí interesa, la toma de decisión. La autora afirma que ciertos estudios han comenzado a redirigir sus enfoques poniendo énfasis en las personas más que en los medios de transporte. Esto supone la superación de observaciones cuyo sesgo materialista destaca la movilidad y el transporte en tanto atributos de la satisfacción de necesidades y deseos, más que valores en sí mismos. Mientras el transporte es el medio o vector que realiza el desplazamiento y la oferta de infraestructuras y servicios, sostiene
Gutiérrez, la movilidad es una performance en el territorio, es decir, una práctica social de desplazamiento territorial que incluye aspectos subjetivos, objetivos, materiales e inmateriales, materializables y materializados. Según la autora, el universo de estudio de la movilidad urbana remite a los viajes realizados (unidad de estudio del transporte), pero también a los viajes realizables y concebidos. Sheller y Urry (2006) manifiestan que mientras los estudios tradicionales del transporte distinguen los viajes de las actividades y los viajantes de los lugares, el nuevo paradigma de la movilidad sostiene que las actividades pueden ocurrir también en movimiento, al tiempo que los lugares pueden ser también creados a partir del movimiento. Esta perspectiva permite replantear el modo de concebir las infraestructuras viales más allá de su carácter meramente técnico y funcional, y comprenderlas en tanto sistemas híbridos de materialidades y movilidades que combinan objetos, tecnologías y sociabilidades (Sheller y Urry, 2006). En este sentido, reemplazar el término infraestructura por "lugares o espacios del movimiento" puede ser el primer paso de este replanteo.

Puede decirse que la Avenida Circunvalación Oeste fue concebida, proyectada y construida en el marco del entendimiento que aquí se pretende superar, es decir, en tanto objeto técnico para resolver dos funciones especificas, defenderse del río y circular lo más rápido posible. ¿Pero qué pasaría si a este uso dominante en sentido longitudinal se le superponen usos transversales dirigidos a la conexión entre el río y los barrios del Oeste y, por consiguiente, con la ciudad en general? ¿Será posible transformar el límite que supone la Circunvalación en un espacio del movimiento 
que incluya ambas direcciones longitudinales y transversales y, por lo tanto, convertirse en mecanismo para la integración del borde Oeste? Entendiendo por integración no sólo a la posibilidad de acceso al centro urbano por parte de los sectores marginales, sino también al intercambio e interacción socio-espacial que puede surgir a partir de nuevos espacios públicos. Se trata, por lo tanto, de elevar la presencia física, el contacto e intercambio entre ciudadanos habitantes de distintos barrios de la ciudad, de la revalorización de espacios urbanos a partir del uso colectivo. Sorkin (2014 [1999]) afirma que el modo en que la ciudad construye sus significados se transforma a medida que la presencia física deja de ser el medio principal de participación y disfrute de la vida urbana.

En relación a las vías especializadas, rápidas y segregadas, Peremiquel Lluch y León Vivanco (2017) sostienen que el movimiento en las ciudades ha de buscar el modo de integrarlas al entramado urbano permitiendo conectar las partes que separa. Tras una larga preocupación enfocada en el tráfico y en la reducción de su impacto en las ciudades, los autores manifiestan la importancia de colocar en el centro de la discusión viaria a la parte más débil, al sujeto relegado: el peatón; y vinculan la dificultad de acceso peatonal con la segregación espacial y social, pues "[...] allá donde no se puede acceder peatonalmente la integración urbana es más difícil" (Peremiquel Lluch; León Vivanco, 2017, p. 6). Así, el papel exclusivo para el peatón junto a la integración urbana, se convierten en objetivos centrales del debate de los espacios de la movilidad.

Ahora bien, en relación a las políticas urbanas contemporáneas la movilidad se ha convertido en tópico frecuente en el ámbito académico $y$, fundamentalmente, en la agenda de los gobiernos locales, a partir del diseño de planes de movilidad urbana y/o intervenciones parciales para el ordenamiento del transporte. No obstante, en muchos casos la problemática de la movilidad es introducida de manera superficial, ya sea con el objetivo de construir una imagen urbana de vanguardia, convertida en una suerte de snobismo político o en una pseudonecesidad como política urbana (Nuñez y Roze, 2014); o bien, ante la ausencia de herramientas teóricas y metodológicas para el abordaje del problema en profundidad. La movilidad está de moda, y la generalización y reproducción acrítica de nociones como movilidad sostenible o sustentable, movilidad inteligente, movilidad en bicicleta, ciudad en movimiento, etc. Ha derivado en anclajes empíricos parciales y sesgados; al tiempo que el fetichismo construido en torno al concepto de movilidad lo convierte en obstáculo epistemológico en el proceso de conocimiento de la realidad (Nuñez y Roze, 2014). Y, al mismo tiempo que se experimentan éstas imposturas y/o deficiencias por parte de los gobierno locales, la movilidad en tanto mecanismo de integración dirigida a sectores urbanos vulnerables y segregados no es sino relegada y omitida de las políticas urbanas.

Ante esta normalización de problemáticas urbanas y su consecuente traducción en líneas hegemónicas de acción programáticas, planes y/o políticas, Nuñez y Roze (2014) sostienen un modo alternativo de búsqueda de explicación de la realidad a la que denominan "crítica conceptual". Esta propuesta apunta, entre otros aspectos, a convertir en observable la génesis social del problema y en recuperar 
la historicidad de las políticas urbanas implementadas sobre el mismo, evitando la homogeneización de los procesos sociales que oculta las particularidades socio-territoriales y las causas estructurales. A continuación, se analiza el proceso de construcción del borde Oeste y su relación con el trazado de la Avenida Circunvalación y la costa del Río Salado, a partir de los planes urbanos confeccionados para la ciudad de Santa Fe a lo largo del siglo $X X, y$, por lo tanto, del pensamiento urbano subyacente a las políticas urbanas implicadas en el problema de la movilidad.

\section{La construccion historica de la inmovilidad en el borde oeste de la ciudad de Santa Fe: lecturas a partir de planes y documentos urbanísticos}

La utilidad de analizar ciertas problemáticas urbanas, en este caso el par movilidadinmovilidad, recuperando la historicidad de documentos y planes urbanísticos confeccionados en un contexto determinado, radica en la comprensión de los fundamentos que subyacen a la construcción del entorno urbano a lo largo del proceso de urbanización. Muchos de los planes que se analizarán a continuación no alcanzaron el nivel jurídiconormativo y sus propuestas apenas se tradujeron es esbozos imprecisos; no obstante, interesa aquí la reconstrucción del modo en que fue pensado el margen Oeste y su influencia en la formación de inmovilidades urbanas asociadas a procesos de segregación y desigualdad social.
La demarcación de los primeros límites urbanos y la aplicación de teorías higienistas

Hacia finales del siglo XIX, el margen Oeste de la ciudad de Santa Fe comenzó a formar parte del ideario urbano y de las propuestas para estructurar la periferia. Entre los proyectos de ordenanzas del Intendente Juan Arzeno elaborados en 1889 y presentados al Concejo Deliberante, ${ }^{2}$ el cierre de la ronda de bulevares planteó un límite a la urbanización Norte y Oeste a partir del trazado del Bv. Gálvez y Bv. Zavalla, respectivamente (Figura 3). Si bien existe cierta voluntad por dotar de lugares de interés a la ciudad y de generar una trama de espacios colectivos (Collado, 1994, p. 15), la relación del tejido urbano con la costa del Salado aún no está siquiera planteada, y la misma noción de "cierre" manifiesta de manera incipiente un rechazo que luego será reproducido a lo largo del siglo XX. Al tiempo que el margen Este sobre la costa de la Laguna Setúbal se convertía en objeto de intervención para la aplicación de teorías urbanísticas asociadas al embellecimiento de las ciudades, a través de la construcción del Parque Oroño y la avenida costanera, se afianzaba una imagen postergada del crecimiento Oeste. En la estrategia de formalizar bulevares de ronda que bordeen el centro consolidado, se prevé la instalación de los principales equipamientos sanitarios de la ciudad sobre el Bulevar Zavalla, proyectando hacia el Oeste una funcionalidad específica relacionada con la salubridad pública. Funcionalidad que rápidamente se materializó con la construcción del Hospital 
Figura 3 - Reconstrucción cartográfica de las propuestas del Intendente Juan Arzeno entre 1887 y 1889

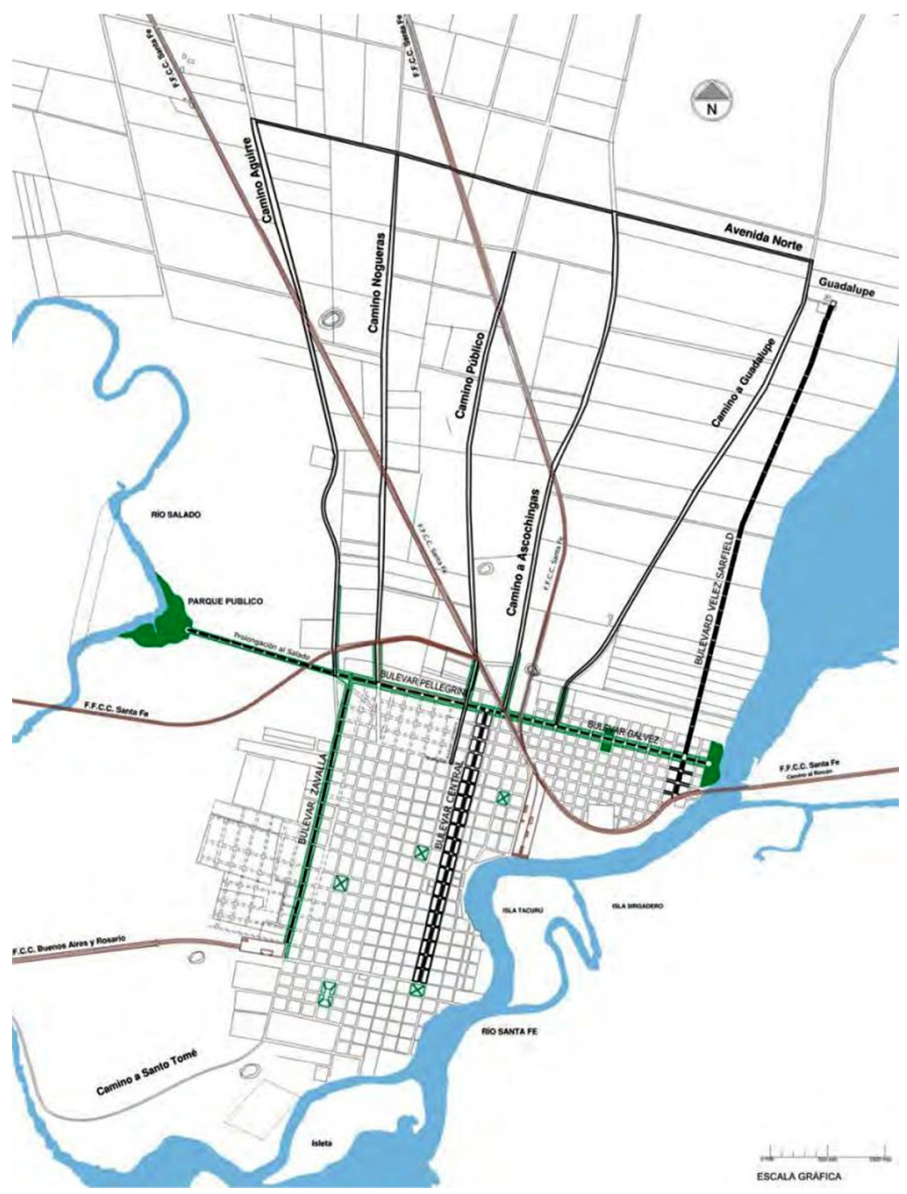

Fuente: Collado (2007)

Italiano, la Casa de Aislamiento, el Asilo de Mendigos y el Hospital de Caridad.

Algunos años más tarde, entre 1908 y 1912 el intendente Edmundo Rosas presenta ante el Concejo Deliberante y la prensa local una serie de proyectos para reconfigurar la planta urbana de la ciudad de Santa Fe. Las propuestas, que según Collado (2007) pueden ser entendidas como un verdadero plan urbanístico, focalizarán nuevamente en la costa Este de la ciudad. Precisamente en los terrenos ganados al río a partir de la 
Figura 4 - Reconstrucción cartográfica de las propuestas del Intendente Rosas entre 1908 y 1912

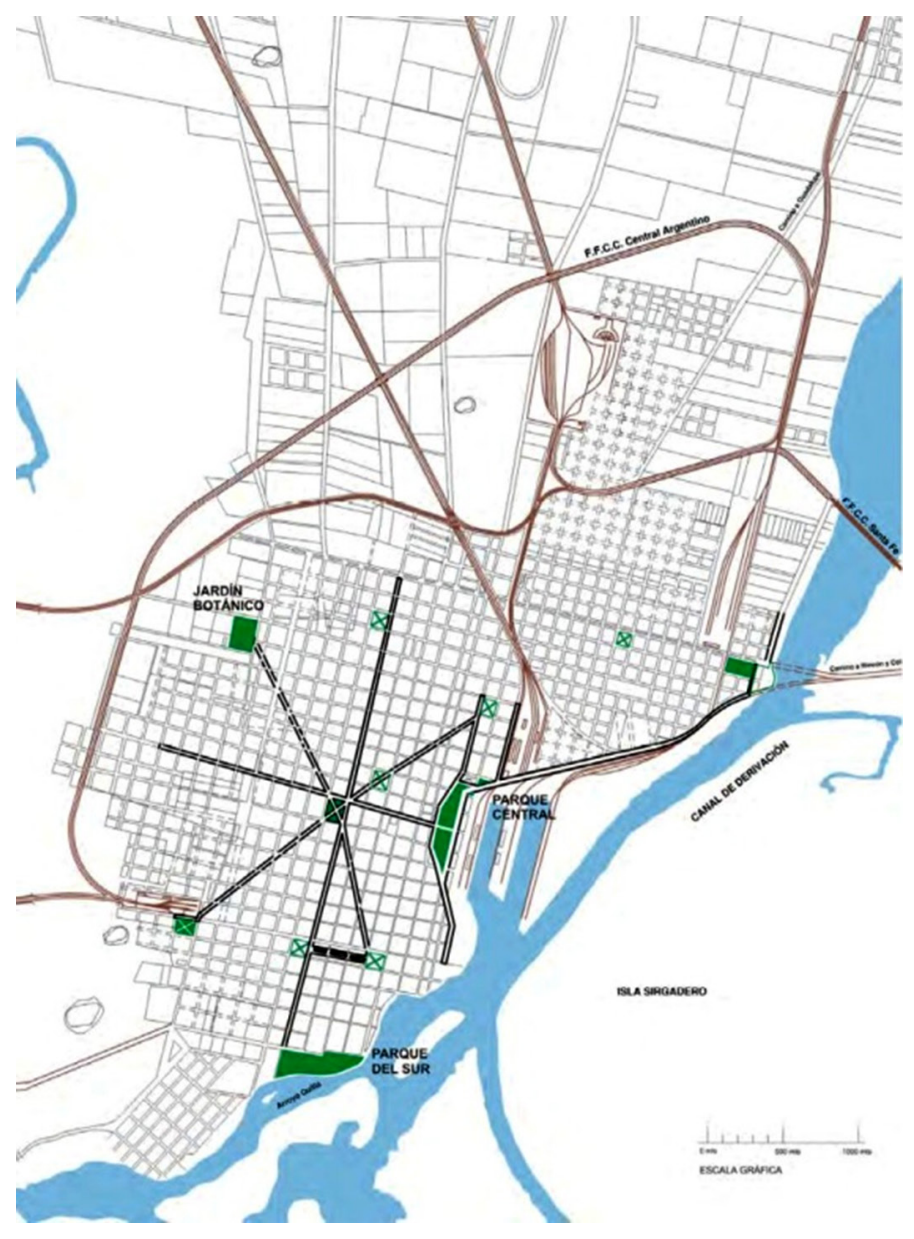

Fuente: Collado (2007).

construcción del Nuevo Puerto de Ultramar, donde se proyectó la instalación del Parque Central, una avenida diagonal hacia el norte y en vinculación con el Parque Oroño y el ensanche de la Avenida Rivadavia. Si bien el plan global planteaba un sistema de avenidas ortogonales y diagonales junto a la ubicación estratégica de parques urbanos, es evidente que la relación de la ciudad con el Río Salado aún no representa un motivo de interés a ser incorporado en el ideario urbano (Figura 4). 
El modelo racional de planificación en Santa Fe: criterios de zonificación y la conformación de un borde subordinado

Por iniciativa del concejal Julio Cabal, en diciembre de 1927 se presentó ante el Concejo Municipal el primer plan urbano para la ciudad de Santa Fe. El denominado "Plan de Urbanización" consistió en un conjunto de iniciativas dirigidas a regular el desarrollo urbano en términos estrictamente funcionales y estéticos. A partir de criterios distributivos y de zonificación, un grupo de propuestas abogaban por la localización de barrios tipo jardín y de nuevos espacios verdes sobre el margen de la Laguna Setúbal, mientras que la previsión del área industrial, los grandes contenedores, los cuarteles y barrios obreros fueron planteados hacia el margen Oeste próximo al Salado, sentando las bases de un ideal fuertemente segregado espacial y socialmente (Figura 5). Esta voluntad por diferenciar zonas urbanas, se manifiesta en la descripción del trazado de la Avenida Circunvalación:

Figura 5 - Reconstrucción cartográfica de Plan de Urbanización de 1927

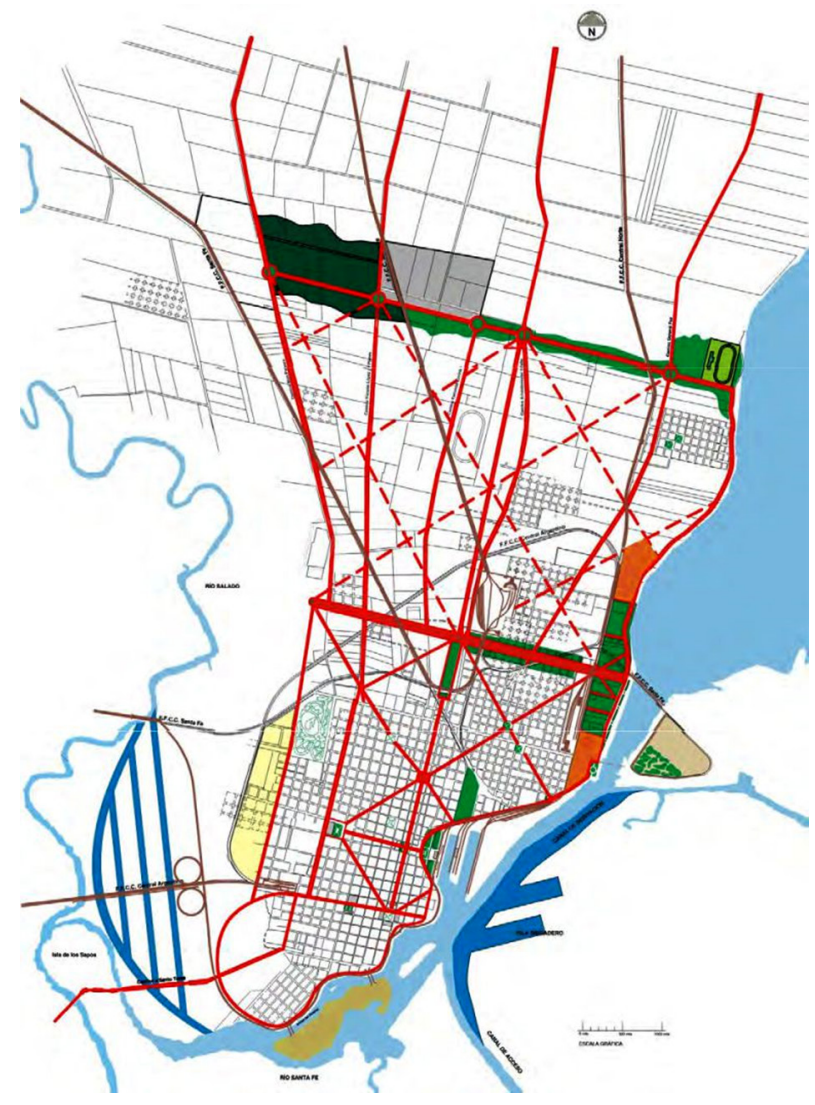

Fuente: Collado (2007). 
Esta avenida arrancará del final de la avenida República, pasará delante de los grandes parques urbanos, de los barrios parques destinados a la edificación costosa y bella, continuará por delante del pueblo de Guadalupe, seguirá haciendo curvas de hermosa perspectiva a la Laguna Setúbal, cortará perpendicularmente el eje del gran Stadium y Gimnasio Municipal, y, abandonando la laguna se dirigirá hacia el Oeste, después, contorneando la extensión posible de la ciudad se dirigirá al Sud, para pasar lateralmente por la zona industrial y por delante de los barrios obreros hasta alcanzar la proyectada avenida 27 de Febrero. (Collado, 2007, p. 590)

Si bien el plan focaliza en los problemas vinculados a la circulación y propone un sistema de vías diagonales que permitirían el acceso a toda la planta urbana, la propuesta de una avenida costanera de carácter paisajístico sobre el margen Este, no es siquiera planteada a lo largo del margen Oeste, considerando que hacia la fecha una buena parte del crecimiento urbano se consolidaba en esa dirección. A pesar de quedar sin efecto, la idea manifestada en el plan de trasladar el área industrial, de servicios y de almacenamiento, nutrió no sólo el ideario de futuros documentos urbanísticos. La construcción material y simbólica del borde Oeste estuvo ligada a sucesivas connotaciones negativas, a partir de la instalación de diferentes equipamientos urbanos considerados indeseables e insalubres para el desarrollo de la vida urbana, a saber: matadero, lazareto y asilo para inmigrantes, cementerio, cárcel, reformatorio de menores, hospital psiquiátrico y hospitales.
Casi veinte años más tarde de la publicación del primer plan urbano, se presenta en 1947 el Plan Regulador de la ciudad de Santa Fe, por intermedio del Instituto Argentino de Urbanismo y el arquitecto y urbanista Miguel Roca. Nuevamente, y con mayor énfasis, la zonificación y separación de actividades constituye el criterio fundamental en el plan de ordenación funcional, donde los terrenos baldíos ganados a los bañados del Río Salado vuelven a ser considerados óptimos para la localización de la zona industrial (Figura 6). Así, la caracterización periférica del margen Oeste y la incompatibilidad de los usos allí proyectados respecto del resto de las actividades urbanas, refuerzan la imagen denostada de la ribera occidental:

La zona industrial quedará en esa ubicación lo suficientemente próxima a la ciudad como para poder servirla sin inútiles estorbos, y lo bastaste bien colocada como para no contaminar el aire que respiran sus habitantes [...] Ha de prohibirse en la zona industrial todo edificio destinado a habitación, salvo el caso bien justificado de residencia para el personal cuya presencia sea imprescindible en el establecimiento fuera de las horas normales de trabajo. (Roca, 1947, p. 137)

Entre las preocupaciones del plan, los problemas urbanos causados por el entramado de las vías ferroviarias ocupan un lugar central en la propuesta. Con el objetivo de liberar la planta urbana de esa "intricada ramazón", de esa "tela de araña perniciosa y fatal", se plantea el emplazamiento de la estación central en la zona Oeste sobre los terrenos ganados a los bañados del Río Salado. La connotación negativa que recibe nuevamente el borde 
Figura 6 - Reconstrucción cartográfica del Plan Regulador Arq. Miguel C. Roca, 1947

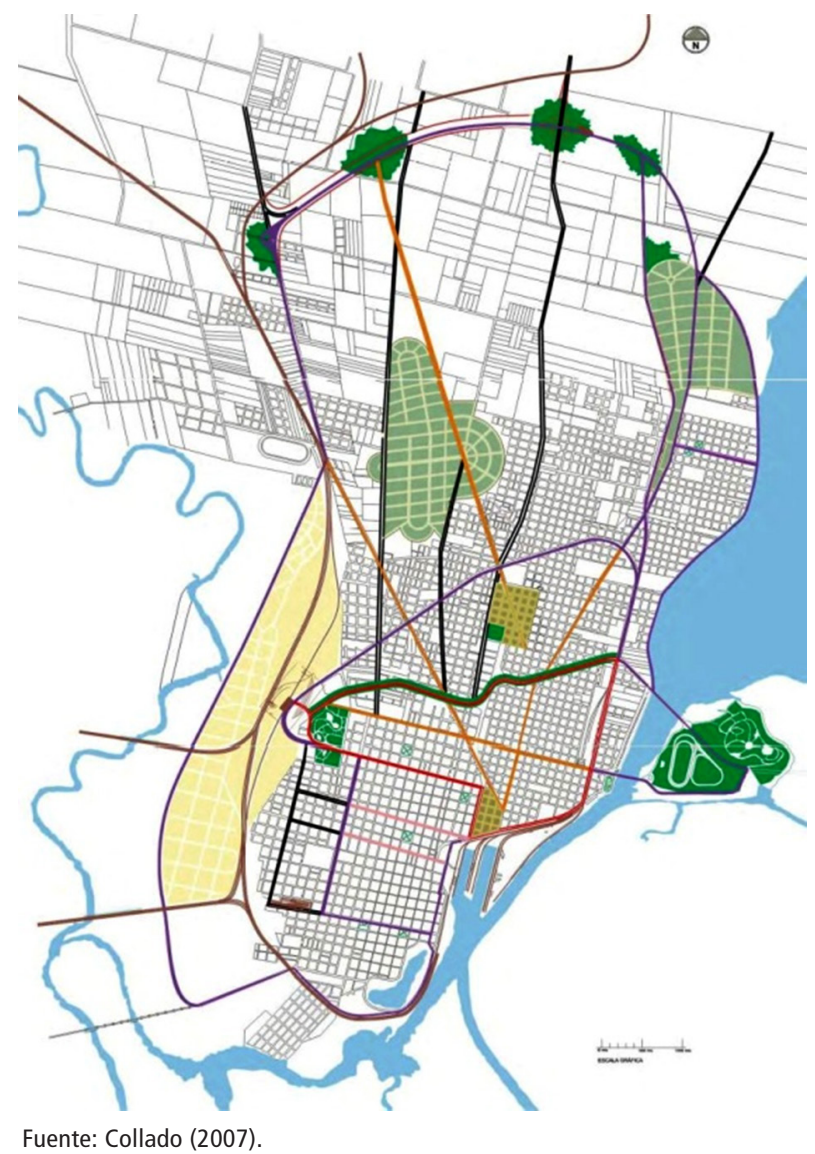

Oeste queda manifestada en la voluntad por convertir en bellas avenidas las zonas que hasta el momento ocupaban las líneas férreas, y en modernos parques y jardines los lugares ocupados por las estaciones; al tiempo que el espacio destinado a la nueva localización se mantiene alejada de los preceptos defendidos en el plan en cuanto a la higiene, el confort, la belleza y la seguridad social:

Por otro lado, esta región tiene la ventaja de estar estrechamente unida a la futura zona fabril de la ciudad, permitiendo servir sus industrias con cortos ramales férreos que no provocan ninguna interrupción de transito, con el agregado que los vientos dominantes llevan miasmas, emanaciones y las humaredas de trenes y talleres, hacia zonas que no pueden perjudicarse. (Roca, 1947, p. 99)

Algunas décadas más tarde, la Dirección de Planeamiento Urbano y Proyectos de la Municipalidad de Santa Fe sanciona y promulga la Ordenanza 7871 correspondiente al Plan Director del año 1980, desde el cual se formula en 1986 el primer Plan de Zonificación para la 
ciudad. En relación a las propuestas anteriores, existe en este plan un abordaje integral que involucra por primera vez las áreas periféricas, a partir de un enfoque enmarcado en una serie de planes con voluntad de regionalización (Rausch, 2010). No obstante, el tratamiento del margen Oeste mantuvo la subvaloración que el sector había recibido en tanto área de servicios. Allí donde los planes del '27 y '47 preveían la localización de la zona industrial, el Plan del 80 plantea, al igual que en el Plan de Roca, la ubicación de la central única de trasporte en el marco del proyecto de sistematización ferrourbanística y rectificación del Río Salado; al tiempo que la zona industrial se traslada hacia el Norte próxima al ejido municipal y aledaña a la costa del Salado (Figura 7).

\section{Planificación estratégica} y perspectivas incipientes de integración del borde Oeste

En consonancia con el auge de la planificación estratégica en el ámbito urbano, el municipio de la Ciudad de Santa Fe presenta en el año 2007 un nuevo plan denominado "Santa Fe 2010. Visión de la Ciudad y Plan Urbano". Puede decirse que, entre los planes confeccionados para la ciudad, es la primera propuesta en considerar el borde Oeste desde su potencial paisajístico. El modelo territorial planteado implica la implementación de una serie de proyectos estructurales, definidos de acuerdo a programas de intervención

Figura 7 - Plan Director de 1980. Planta General

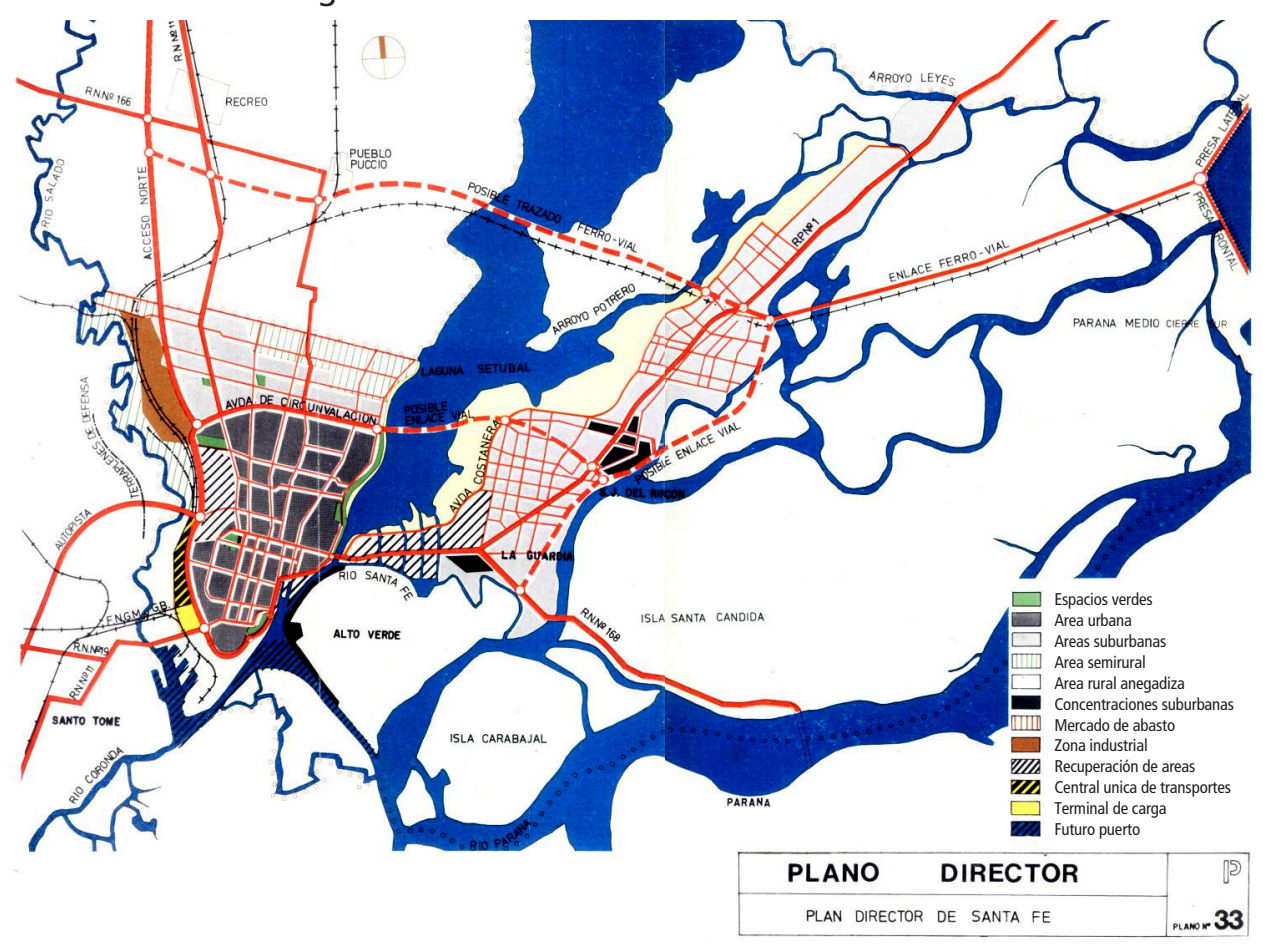

Fuente: Municipalidad de la ciudad de Santa Fe (1980). Plan Director Ciudad de Santa Fe. Santa Fe, MCSF. 
sobre grandes sectores de Ciudad. Los mismos corresponden a: Corredores Centrales de Articulación Interna, Corredores de Articulación Ciudad-Río, Corredores Transversales de Articulación Interna EsteOeste, Frente Norte, Áreas de Desarrollo Productivo y Articulación Metropolitana y Propuesta Ferroviaria. La recurrencia a la idea de corredores queda manifestada en el plan a partir de su definición:

El concepto de corredor se refiere a un espacio público que organiza una secuencia de actividades productoras de centralidad y de atracción a inversiones comercio, servicios, equipamiento público, actividades productivas de pequeña 0 media escala- dispuestas a lo largo de ejes circulatorios que articulan espacios públicos y residencias, y que funcionan como motores del desarrollo de los sectores urbanos. (Municipalidad de la ciudad de Santa Fe-MCSF, 2007, p. 222)

En el marco de los Corredores de Articulación Ciudad-Río, el proyecto del Corredor del Oeste "invita a prestar atención a un frente con ricas potencialidades urbanas y paisajísticas" (MCSF, 2007, p. 222), a partir de un mayor compromiso con la reconstrucción social de la ciudad luego de la catástrofe hídrica del año 2003 (Figura 8). Se trata, por lo tanto, de revalorizar el Río Salado en tanto paisaje y mitigar las condiciones inseguras del hábitat a través de la generación de un sistema de espacios públicos, incrementando la accesibilidad y la conectividad con las aéreas centrales. En este sentido, puede decirse que subyace a este planteo la idea aquí defendida en torno a la movilidad como mecanismo de integración, una movilidad multidireccional que intenta revertir la inmovilidad asociada a sectores urbanos considerados inaccesibles para el imaginario colectivo del resto de los habitantes.

El último plan con el que cuenta la ciudad de Santa Fe corresponde al Plan Urbano del año 2009, del cual deriva el Reglamento de Ordenamiento Urbano (ROU) vigente a la actualidad. Al igual que en los planes de 1980 y 2007, existe una lectura y diagnostico de las aéreas vulnerables y marginales en continuo crecimiento sobre el borde Oeste. Sin embargo, la problemática vuelve a reducirse a criterios de estricta zonificación y con débiles planteos de integración, intercambio y revalorización de la ribera Oeste. Entre las áreas de planificación especificadas en el ROU, los principales distritos del borde Oeste se zonifican de acuerdo a los siguientes usos: I (Industrial); R3 (Residencial de baja densidad en proceso de integración); R5 (Residencial de baja densidad en área en consolidación); ZSH (Zona de Seguridad Hídrica); RUA (Rural Anegadizo). No obstante ello, cabe destacar una visión de apertura y búsqueda de reconversión del borde Oeste a partir del proyecto Reserva Natural Urbana y Gestión de Riesgos Climáticos, aprobado según Ordenanza Municipal 12179 (Figura 8). El mismo consiste en la transformación de los reservorios del sistema de drenaje urbano en un espacio verde de uso público y atractivo paisajístico. Este planteamiento se enmarca en una política de gestión de riesgo desarrollada desde el año 2008 y en la implementación de la idea de ciudad resiliente.

Pese a estos intentos por revertir el imaginario del borde Oeste, construido históricamente como un margen subsidiario y de servicios, la culminación de la Avenida 
Circunvalación en el año 2012 reforzó la barrera entre ciudad y río, negando el paisaje costero y jerarquizando la movilidad vehicular rápida. Este desfasaje entre la concreción de obras públicas de escala territorial y el ideario urbanístico, denota la ausencia de planes integrales de desarrollo urbano y la escasa coordinación entre las distintas escalas gubernamentales, como así también al interior de los gobiernos locales.

Figura 8 - (Izquierda) Proyecto Corredor del Oeste (Derecha) Proyecto Reserva del Oeste

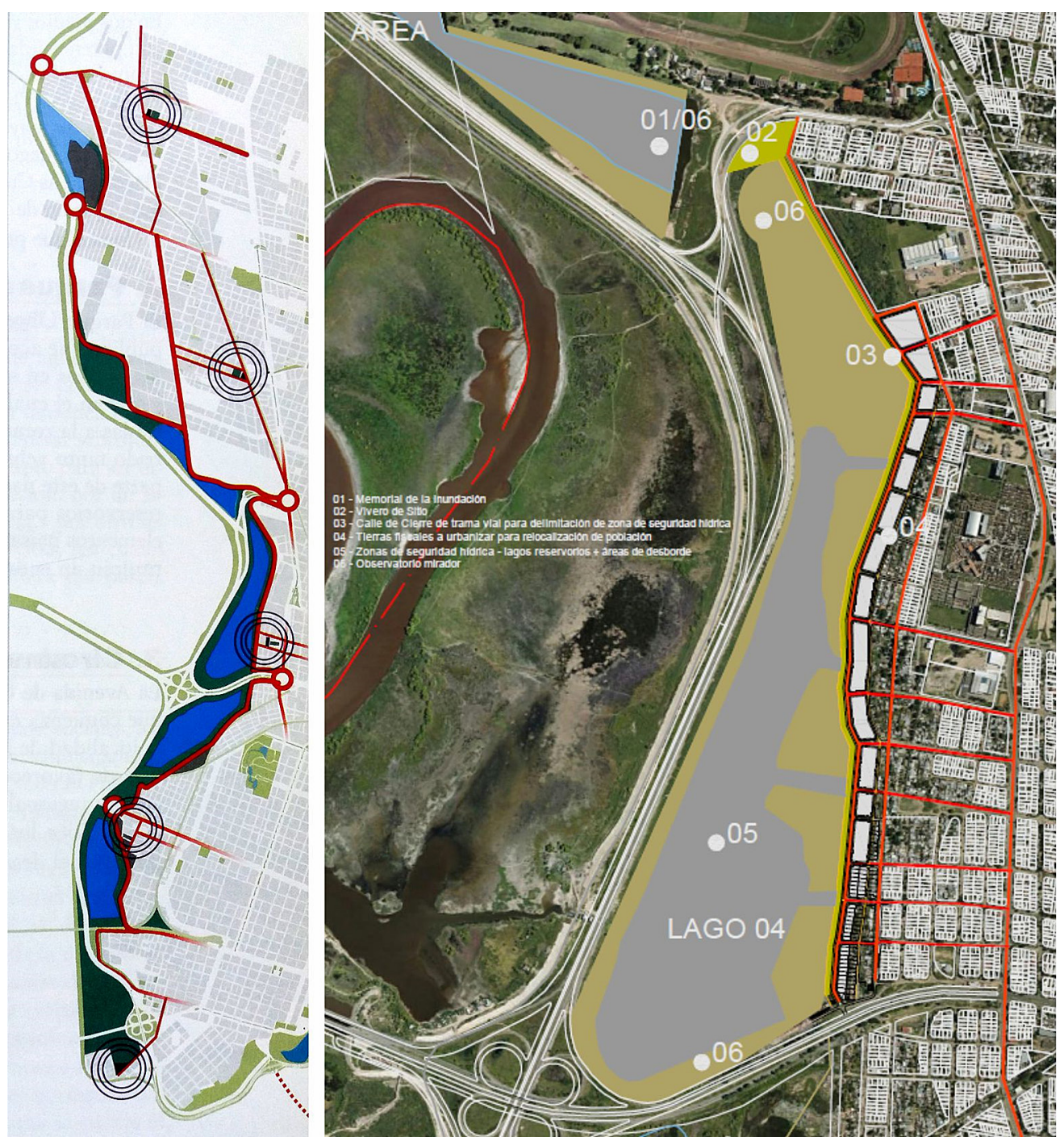

Fuente: (Izquierda) Municipalidad de la ciudad de Santa Fe (2007). Santa Fe 2010. Visión de la Ciudad y Plan Urbano. (Derecha) Proyecto Reserva del Oeste. Fuente: recuperado de http://santafeciudad.gov.ar/blogs/reserva. 


\section{Consideraciones finales}

Con el objetivo general de comprender los procesos de crecimiento urbano en sus manifestaciones desiguales y fragmentadas, se analizó el proceso de ideación y planificación a través de los diferentes documentos urbanísticos y planes urbanos confeccionados para la ciudad de Santa Fe. A partir de una perspectiva focalizada en las movilidades e inmovilidades urbanas, la lectura se concentró en las propuestas y proyectos ideados en torno al margen Oeste y en su construcción material y simbólica en tanto borde postergado y denostado por las políticas urbanas. Desde la demarcación de los primeros limites que proponían un cierre a la planta urbana hacia finales del siglo XIX y hasta la concreción de la Avenida Circunvalación en el año 2012, la planificación local parecería haber quedado suspendida en el tiempo manteniendo los mismos supuestos por más de un siglo y, fundamentalmente, la misma relación con la ribera del Río Salado.

Las propuestas contemporáneas, precisamente aquellas desarrolladas luego de la catástrofe hídrica del año 2003, replantean el posicionamiento del Río Salado con intenciones de apertura e integración. Sin embargo, las políticas enfocadas en la planificación urbana aún sostienen criterios de estricta zonificación, y no han logrado al momento articular con políticas públicas de movilidad y transporte. En el marco del nuevo paradigma, la movilidad se convierte en mecanismo de integración al ofrecer múltiples movilidades de tipo material, simbólico y cultural, revirtiendo la posición de aislamiento e impenetrabilidad que suponen los barrios del margen Oeste y, por lo tanto, su inmovilidad. Es tiempo de superar el dualismo victima-victimario emergente de la relación infraestructura vial - espacios cotidianos, y redirigir las propuestas y acciones hacia una mayor cantidad de movimientos transversales en los espacios de la movilidad.

\section{Estefanía Szupiany}

Universidad Nacional del Litoral, Instituto de Humanidades y Ciencias Sociales del Litoral, Consejo Nacional de Investigaciones Científicas y Técnicas. Santa Fe, Argentina.

eszupiany@gmail.com 


\section{Notas}

(1) La catástrofe hídrica del año 2003 fue el resultado de un desborde extraordinario del Río Salado que afectó a más de un tercio de la población. La magnitud del evento se tradujo en la pérdida de vidas humanas, un sinnúmero de evacuados y grandes daños materiales.

(2) Los distintos proyectos de ordenanzas fueron presentados aisladamente sin configurar un plan general para la ciudad. Sin embargo, afirma Collado (2007), las distintas propuestas pueden ser leídas de manera articulada dada su proximidad cronológica y la repetida voluntad por sistematizar el área urbanizada, marcar los límites y estructurar la periferia.

\section{Referencias}

COLLADO, A. (1994). Santa Fe. Proyectos urbanísticos para la ciudad 1887-1927. Documento de trabajo n. 2. Santa Fe, Universidad Nacional del Litoral.

(2007). Modernización urbana en ciudades provincianas de Argentina. Teorías, modelos y prácticas, 1887-1944. Tesis de Doctorado. Sevilla, Universidad Pablo de Olavide.

GUTIERREZ, A. (2009). Movilidad o inmovilidad: ¿Qué es la movilidad? Aprendiendo a delimitar los deseos. En: CONGRESO LATINOAMERICANO DE TRANSPORTE PÚBLICO Y URBANO, XV. Buenos Aires.

(2012). ¿Qué es la movilidad? Elementos para (re)construir las definiciones básicas del campo del transporte. Bitácora Urbano Territorial. Bogotá, v. 2, n. 21, pp. 61-74.

MiRALLES-GuASCH, C. y CEBOLLADA, A. (2003). Movilidad y transporte. Opciones políticas para la ciudad. Madrid, Fundación Alternativas.

MUNICIPALIDAD DE LA CIUDAD DE SANTA FE (1980). Plan Director Ciudad de Santa Fe. Santa Fe, MCSF. (2007). Santa Fe 2010. Visión de la Ciudad y Plan Urbano. Santa Fe, MCSF. (2009). Plan Urbano. Santa Fe, MCSF.

NOVICK, A. (2012). "Los proyectos territoriales en perspectiva". En: AAVV. Planes, proyectos e ideas para el Área Metropolitana de Buenos Aires. Buenos Aires, CPAU.

NUÑEZ, A. y ROZE, J. (2014). Las palabras y las cosas en la ciudad latinoamericana. Obstáculos epistemológicos en políticas urbanas argentinas. Cadernos Metrópole. São Paulo, v. 16, n. 31, pp. 61-88.

PEREMIQUEL LLUCH, F. y LEÓN VIVANCO, M. F. (2017). La movilidad no es una moda. QRU: Quaderns de Recerca en Urbanisme. Barcelona, n. 7, pp. 5-7.

RAUSCH, G. (2010). El plan del 80 en Santa Fe: regionalización tardía ante una suburbanización inminente. Cuaderno Urbano. Espacio, Cultura, Sociedad. Resistencia, v. 9, n. 9, pp. 111-129.

ROCA, M. (1947). Plan Regulador de la ciudad de Santa Fe. Buenos Aires, Instituto Argentino de Urbanismo. 
SHELLER, M. y URRY, J. (2006). The new mobilities paradigm. Enviroment and Planning A. Vancouver, v. 38, n. 2, pp. $207-226$.

SORKIN, M. (2014[1999]). “EI tráfico en democracia”. En: MARTIN RAMOS, A. (ed.). La calle moderna en 30 autores contemporáneos y un pionero. Barcelona, Universitat Politecnica de Catalunya.

\section{Diarios y revistas}

EL LITORAL (2012). Circunvalación Oeste: 10 minutos de alto riego. Santa Fe, 27 de Junio.

EL LITORAL (2014). Vandalismo contra autos en la Circunvalación Oeste y la autopista a Rosario. Santa Fe, 3 de Octubre.

EL LITORAL (2017). Fuego en la Circunvalación Oeste. Santa Fe, 14 de Agosto.

EL LITORAL (2017). Reiterados ataques a los automóviles en la Ruta n. 70. Santa Fe, 5 de Septiembre. LT10 UNIVERSIDAD (2017). Le tiraron un palo en la circunvalación para robarle la moto. Santa Fe, 7 de Abril.

\section{Fuentes de Internet}

http://santafeciudad.gov.ar/blogs/ciudad-resiliente/

http://santafeciudad.gov.ar/blogs/reserva/

Texto recebido em 15/set/2010

Texto aprovado em 27/dez/2017 